\title{
Ensembles of Sonic Skills: Conclusions
}

\begin{abstract}
This chapter combines a diachronic with a synchronic approach. It explains how different ensembles of sonic skills, or sets of sonic skills in specific settings, come to prevail with shifting relations between science and technology, science and the professions, and science and society. These ensembles reflect the significance of timing, trust, and accountability in the dynamics of science.
\end{abstract}

Keywords Ensembles of sonic skills - Science dynamics - Timing in the sciences - Trust in the sciences - Accountability in the sciences

\section{INTRODUCTION}

This final chapter builds on the previous ones by showing that listening modes and sonic skills in the sciences come in ensembles. By this, I mean that it is in particular configurations - in recurrent modes and with specific tools - that listening for knowledge has been considered useful or even vital to science, medicine, and engineering. "Ensembles of sonic skills," then, are not simply sets of sonic skills, but also the settings within which sonic skills are appropriated. These ensembles will be discussed here in terms of family resemblances: although the sets and settings may differ in detail, they have certain characteristics in common. The association with musical ensembles is no coincidence. In music, the terms string quartet or wind quintet do not only denote particular combinations of

(C) The Author(s) 2019

K. Bijsterveld, Sonic Skills, https://doi.org/10.1057/978-1-137-59829-5_6 
musical instruments, but also the repertoires they play. And ensembles are not likely to perform on any conceivable occasion-some occasions are more suitable than others. It is set and setting together that make the difference.

Thinking about ensembles will help us to understand, at a more structural level than in the previous chapters, where and why sonic skills survived or returned, as well as where and why sonic skills lost their relevance. Whereas the preceding chapters were either synchronic or diachronic in their approach, this one is synchronic in identifying ensembles of sonic skills, but diachronic in analyzing the scientific dynamics in which they operate. I aim to show how the changing relationships between science and technology, science and the professions, and science and society both enable and constrain the usefulness and legitimacy of listening for knowledge in science, medicine, and engineering. Such shifts are best captured by focusing on issues of timing, trust, and accountability. Scientists and professionals, the topics they examine, the skills and tools they use for engaging their ears, and the wider public all need to be taken into account if we are to understand what sound and listening "do" in the sciences.

In this undertaking, I also hope to slightly revise the historiography of the position of music in the sciences, and to complicate two existing accounts about the senses in the sciences: the claim that scientists sought out to listen whenever the subject under study was not directly accessible, and the emphasis on the significance of immutable mobile, visual inscriptions for the sciences. But let me first address the relevance of ensembles of sonic skills, and how these ensembles cut across the diversity of instances of listening described in the previous chapters.

\section{Timing-And the Relations Between Science AND TeCHNOLOGY}

A widely accepted, Latourian argument for the dominance of visualized presentations of data in science has been their immutability and their convenient combinability with texts on paper and screens, which contributes to the easy circulation of scientific claims. I have referred to this several times: scientific inscriptions can be synoptically organized and mapped onto each other. That option has also been opened to sound, in synaural form, by the rise of digital sound technologies. MP3 files are now easily combinable and distributable, helping to stimulate the rise of sonification. 
This line of reasoning puts most of its faith in processes of publishing in the sciences, as it extends the networks of "acting at a distance." Yet oral presentations are still crucial elements of doing science, medicine, and engineering, whether at international conferences, in departmental seminars, or for teaching, with or without gestures in the air or the use of black- and whiteboards. Moreover, as we have discussed in Chapter 2, sound is highly compatible with oral presentations. Alexandra Supper $(2015,2016)$ made this point when she discussed the phenomenon of "data karaoke" in the sonification community. When presenting their data at conferences or meeting up with domain scientists, sonification specialists frequently sing their data as they speak about their findings. In doing so, they are embodying their data, often combining them with gesticulation and using the voice to highlight particular aspects of their results, and thus of their analysis. This is especially helpful when working with domain experts, who are usually less well versed in diagnostic listening to sonifications. As an additional advantage, users can trust their own voice rather than run the risk of sound files or speakers failing at the critical moment, for instance because they are not connected properly or otherwise out of order. Voices and gestures are easily available, any moment, anywhere. Similarly, other forms of embodied representation survived because it enabled practitioners to The choice of a particular sensory modality thus depends both on compatibility with the modalities already dominating the setting and on the importance of timing a particular action in that setting.

Equally, opinions as to whether or not particular tools, such as the phonograph or the magnetic tape recorder, are appropriate in a particular domain of science may be guided by issues of timing. In the world of naturalists sound-hunting for easily disturbed birds, for instance, the cumbersome combination of phonograph and wax heaters was not immediately taken up. Magnetic tape recorders, in contrast, embodied the kind of portability welcomed in the field. Even in the analysis of a visual inscription, the sound spectrogram (assumed to be efficient for swiftly surveying results), some believed an auditory approach could contribute to the analysis, for instance by creating crude classifications by ear before zooming in on the details. Again, in these situations, the need for careful timing of a particular action in the process of doing science informed the choice of one particular sonic instrument over another, or the decision to start with listening before focusing on visual inspection. 
In this essay's second chapter, I also pointed out the significance of urgency-the imperative need to take action at a particular moment in time-for the survival of verbal expressions or manual notations of sound. When teaching medical students or tracking birds in the field, such ways of capturing sound may be the most efficient strategy for recording data or communicating knowledge on the spot. Similarly, the stethoscope survived in situations where high-tech instruments were not available or where urgency reigned, such as in wartime hospitals or countries unable to access the full range of expensive diagnostic instruments. Indeed, even in high-tech contexts stethoscopes may be of use in urgent situations, as a story recounted by European Space Agency cosmonaut André Kuipers illustrates. Kuipers, a Dutch physician by training, worked with a Russian aerospace engineer, the commander, and an American chemical engineer on board the International Space Station (ISS) for over six months in 2011-2012. One day, a space capsule arrived with new and essential supplies. Once the capsule had docked to the station, the commander made rotating movements to open the door. But whatever he tried, the door remained closed. He and his colleagues had no clue what might be causing the problem. It was in this setting that Kuipers' identity as a doctor came in useful, and led him to his stethoscope-in order to listen to the door. This enabled him to work out whether or not there was a mechanical issue. The clicks and sounds of rotating parts told him that the door itself was not the problem. It simply required the strength of three cosmonauts to open the door. ${ }^{1}$ Kuipers's diagnostic listening skills saved the day in this expedition episode; in a context of urgency, the stethoscope turned out to be a highly efficient instrument.

These observations help me to qualify Sophia Roosth's (2009) suggestion that scientists tend to rely on their ears or on listening tools once they cannot directly access (in her examples: see) the phenomena of their interest. This is not the full story of why scientists come up with the idea of listening or are ready to explore it. First, prior listening experiences may help scientists to reconceptualize a problem or phenomenon as being open to listening, as the ISS story shows. In another example, the realization of sound's role in survival during World War I paved the way for academics to acknowledge the Geiger counter's usefulness in measuring radiation. Second, the societal relevance of a problem plus the availability of new instruments may also frame it as amenable to a listening approach. It has long been known that icebergs-one of 
the examples with which this essay began-can only be partially seen. What has opened up the underwater portions of icebergs more systematically to the epistemology of listening is the current sense of an urgent climate problem, in combination with the availability of high-quality hydrophones and ways of digitally transmitting sound files. Likewise, early nineteenth-century physicians already had access to percussion as a way of making the body legible; the readiness of many of them to embrace the stethoscope was spurred by a new desire to create physical distance between patient and doctor. Third, for a sound technology to be accepted as an instrument of investigation, it often requires material or conceptual sensory calibration with the sensory modalities of equipment used in the past. And fourth, as so many STS scholars have argued before us, making objects aurally (or visually) researchable redefines the character of those objects themselves-think of what the clean, visually informed sound captured by microphones with parabolic reflectors meant for definitions of and approaches to bird vocalization. All this complicates the idea that as soon as an existing phenomenon cannot be seen, the time has come to start listening.

Returning to the significance of timing one's actions for listening in the sciences, as an ensemble of sonic skills, we should also recall the work of synchronization as a structuring aspect of modern-day laboratories. For facility staff as well as scientists, showing an ability to adjust to the temporalities of planning schedules and instruments' workings expresses their efficiency to their superiors. In Joeri Bruyninckx's study, monitory listening to the instruments, and talking about sound when training newcomers to the lab, was an important dimension of an "embodied awareness of time"(Bruyninckx 2017: 840).

\section{Trust-And the Relations Between the Sciences AND THE Professions}

The extent to which audiences have invested trust, a solid belief in the reliability and responsibility of a person's or institution's actions, in those who listen for knowledge has deeply affected the levels of justification required for experts to rely on their ears. Such audiences ranged from patients and customer-drivers to scientific peers.

When, in the late 1920s, motorists started to question the expertise of the car mechanics who repaired their cars, the existing ensembles of 
sonic skills came under pressure. German mechanics responded both by casting doubt on the listening capacities of their clients and by creating a societally acknowledged jurisdiction for their own trade. They reclaimed the ability to listen diagnostically to engines as their exclusive skill-at the expense of drivers, who were to limit the use of their ears in automotive contexts to monitory listening. At the same time, they successfully established a guild-like system designed to strengthen clients' trust in the mechanic's expertise in diagnostic listening. In the United States, the same repair crisis played out rather differently. As US mechanics did not have Germany's tradition of protected trades, client trust in their sensory skills was easily undermined; that trust was transferred to measuring instruments featuring visual displays. Clients might be just as unable to read these instruments as to understand the mechanic's sonic skills, but the promise of transparency had the required effect.

The German mechanics modeled themselves on doctors, claiming the same type of tacit, clinical expertise and a similarly well-founded authority to describe sounds in their own words. They maintained this auditory autonomy until, with the rise of electronics, automotive technology itself had less to say through sound. In the world of medicine, the distance created by the stethoscope initially enhanced patients' trust in doctors. When medicine had to comply to the same standards of objectivity as science, it moved towards standardization, but at the bedside and in teaching contexts, doctors may still refer to sounds and their diagnostic meanings, in whatever ways they find effective.

Anna Harris has noted that despite the decreasing relevance of auscultation in hospitals and skepticism about its future, students worldwide are still being trained in both auscultation and percussion. This is because senior physicians and medical educators believe that teaching medical students "how to use a stethoscope to find heart murmurs or how to percuss the borders of the liver" is vital for honing their "sensory awareness" (Harris 2015). What is important here, Harris and doctor-educator Eleanora Flynn claim, is the education of students' attention-a notion introduced by James J. Gibson and taken up by Tim Ingold and Bruno Latour. In this "education of attention," learning to listen feeds into learning to pay attention, "a quality that is important for all sensory skills, not only those of auscultation and percussion" (Harris and Flynn 2017: 4). Students learn to notice differences in sensation, a 
skill many medical professionals consider crucial to their own and students' observatory and diagnostic abilities. Even if the skills of percussion and auscultation are on the decline as diagnostic techniques in hospital settings, therefore, training in these skills fulfills a "pedagogical purpose," thereby contributing to students' medical identities-not least through how they practice on, experiment with, and discover their own bodies (Harris 2016: 52).

In labs, sensory awareness is no less important, as an embodied vigilance concerning the material conditions of experiments helps to synchronize organizational and instrumental time. Whereas many doctors and mechanical engineers pride themselves on their sonic skills as much as other problem solving skills, lab technicians tend to make their skills invisible. The technicians observed by Bruyninckx mobilized "valuable social capital" to arrange replacements and free advice in order to keep the systems running. They did so largely out of sight of the lab's management, however, apparently fearing that technicians' visibility would actually highlight "ruptures in the operation they are charged (and paid) to maintain" (Bruyninckx 2017: 840-841). Keeping their skills out of view of those higher in the hierarchy contributes to an impression that everything is running smoothly, paradoxically reinforcing trust in technicians' work, which is not sanctioned by professional jurisdiction in the way achieved by mechanics and doctors. But it also makes the vital importance of monitory listening for contemporary lab life less obvious to STS researchers-unless one specifically focuses, as we have done, on the role of sound in science.

The inverse relationship between trust invested in experts and the justification required to employ sonic skills is perhaps best illustrated by those who are entrusted and hired by governments to display generalized distrust, such as the secret service, police, or military. In those settings, listening to the "enemy" and analyzing sound has never lost its importance, despite these activities having been backed up by visual techniques as well. In turn, those monitored-from soldiers, spies, and criminals to prisoners (Rice 2016) - have availed themselves of nonverbal self-expression and counter-eavesdropping on their sonic environments in order to stay under the radar of surveillance. Clearly, we should not forget that sonic skills are not limited to those working in science, medicine, and engineering (Bruyninckx and Supper 2016: 2), even if these groups have taken center stage in this essay. 


\section{Accountability-And the Relations Between Science AND SOCIETY}

Technicians may have various reasons for carrying out some of their repair work discreetly, "outside the organization's 'visible' range of auditing and accounting tools" such as the billed time of instrument time. They do so "to maintain their professional status, to protect their social capital, or in order not to provoke a further tightening of organizational temporalities" (Bruyninckx 2017: 841). This may, however, render inadequacies in organizational plans or replacement schemes invisible as well and sustain a high tempo of research, potentially to the point of compromising researchers' ability to properly understand, work with, and interpret the instrumental set-up. Synchronization work supported by the senses thus keeps in place the system of accountable time.

The experienced nurses who adjusted the alarms of intensive care instruments to prevent false alerts and unnecessary disruptions to their workflow similarly sidestepped the constraints of accountability. But they did so only temporarily; they also readjusted the alarms to more sensitive settings to ensure that less experienced staff would notice the slightest potential patient problem, and to protect the liability of the hospital and medical instrument manufacturers. In other words, tweaking alarms once again leaves the system of accountability intact. In the lab example, monitory listening in analytic and synthetic modes had to prevent listeners from noticing too little, whereas in the hospital, the senior nurses' interactive monitory listening had to prevent them from noticing too much. In both cases, the accountability structure triggered their use of sonic skills.

Accountability is also relevant for understanding the recent rise of sonification, and especially musical sonification, in the sciences. As explained in the previous chapter, sonification is considered a highly effective tool for attracting the attention of the general public with the promise of an auditory sublime. This close link between science and music seems to contradict the historiography of the evolution of music's position in the sciences. Alexandra Hui studied German-Austrian scholars and scientists examining the sensation of sound between 1840 and 1910. Their laboratory work, she argues, was initially "bound up with musical training and therefore musical aesthetics. Musical skill was a scientific skill" (Hui 2013: 145). Hermann Helmholtz's study of overtones, for instance, compared the workings of the inner ear to those of 
the piano: when striking a particular piano string, strings with corresponding harmonics would resound, and so too would the cortical fibers of the inner ear. Ernst Mach's research on the relationship between sensation and attention also depended on musical examples. He showed that listeners perceived the same sequence of chords quite differently when they focused on either the lower or the upper tones.

By the end of the nineteenth century, however, "the value of musical skill had become contested" (Hui 2013: 145). This was due to a rising interest in non-Western music and a changing musical aestheticssuch as Arnold Schönberg's twelve-tone music, which undermined the dominance of the Western music system-as well as to changing opinions about the ideal experimental listener. In a debate between Wilhelm Wundt and Carl Stumpf over just-noticeable pitch differences, for example, Wundt promoted the use of thousands of listeners, including musically untrained listeners, to substantiate statistically valid claims about sensation. In contrast, Stumpf believed that only listeners with musical expertise could hear the relevant distinctions; the outcomes of studies using musically untrained listeners were invalid. Wundt's approach won the day.

A similar trend seems to have been at work in ornithology as well, as it shifted from musical notation, to graphical notation, to the spectrographic rendering of sound, leaving conventional musical education largely obsolete and enabling contributions by musically untrained scientists. As this essay has illustrated, music did not lose its relevance entirely. Alluring gramophone records of bird sound attracted amateurs, who then contributed to data collection in ornithology. Musical metaphors continued to be used in both the didactics and the analysis of sound. Moreover, some bioacousticians added musical notation to sound spectrograms; others even hired a drum-master to distinguish and identify the rhythmic structures in the inter-click intervals of sperm whales (Harris 2012). And in Harris and Flynn's study, medical students who had received musical training earlier in life thought this helped them to discriminate between bodily sounds and find words for them. As one student explained, talking about lung sounds: "If you play music you listen to a lot of music and you become really particular about ... you hear the phrasing. You can hear the rests, you can hear the pauses. You become more skilled in listening to the nuances of sound" (S7, cited in Harris and Flynn 2017: 4). A musically inspired skills training-focusing on the 
discrimination of rhythm, cadence, pitch, and timbre-enhanced nursing students' ability to notice and interpret the sounds of heart, lungs, and gut, such as the increase of bowel sounds "with diarrhea, gastroenteritis, or early obstruction" (Pellico et al. 2012: 236). In all, however, musical skills have tended to lose their analytic value in the sciences over the late nineteenth, twentieth and early twenty-first century.

How, then, should we understand scientists' currently growing interest in musical sonification and the evocation of the sublime that it promises to deliver? Alexandra Supper has cited the rise of interdisciplinarity in the sciences, noting the explanation of this recent prominence by sociologist of science Andrew Barry et al. (2008). They claim that interdisciplinarity - which in their definition includes the integration of distinct fields of academic scholarship and the integration of science with non-science, such as the arts-draws on three logics: the logic of innovation, the logic of accountability, and the logic of ontology. The idea that the sciences should contribute to technological innovation and economic growth, or the logic of innovation, is now acclaimed within and beyond academia, and has propelled all kinds of research that combines insights and methods from different disciplines to come up with novel medicines or other products for the market. In contrast, the logic of ontology is geared to "effecting ontological change in both the object(s) of research, and the relations between research subjects and objects" (Born and Barry 2010: 105). Reflection on the reality claims behind particular methods, or on the shifting boundaries between humans and technology, pushes academics toward interdisciplinarity, which may then further erode conventional ontologies. The logic of accountability is anchored in the ever greater need to account for the large amounts of public spending on science, and to respond to the declining authority of and a growing public "unease" with scientific institutions. One way for scientists to cope with these pressures is by "enlisting artists" to reach out for audiences who "might develop not only cognitive, but interactive and affective involvements with science" (Born and Barry 2010: 108-109). Interdisciplinary projects of this kind are intended to legitimize investment in and by scientists.

The increasing demand for science's accountability expresses a major shift in the relationships between science and society-a shift that is also an important factor for the rise of sonification projects in which scientists and artists collaborate. But scientists have not been the only ones seeking to enhance the legitimacy of their work through strategic 
alliances. Artists have turned to the sciences with the aim of making their own work more acceptable or visible. This is reflected in the hope expressed by artist John Dunn, mentioned in passing in the previous chapter, of adding "a sense of "deep structure" to music when he worked with biologist Mary Anne Clark on turning protein sequences into a work of sound art (Supper 2014: 43). This two-way strategic referencing phenomenon is known as "legitimacy exchange," a term coined by STS scholar Geof Bowker (1993: 116). The notion indicates how scientists at the margins of particular fields refer to colleagues in other domains of science in order to garner support for their claims. Some sonification projects have even wider ambitions, though. The composition Bonner Durchmusterung by Marcus Schmickler, commissioned for the International Year of Astronomy in 2009, was intended to educate the public about astronomical phenomena such as eruptions of the sun. But the program notes, co-authored by the scientists and artists involved, say that the work also proposes to foster reflection about "the relation between data and the reality of the observed objects" (cited by Supper 2014: 44)-reminding us of the logic of ontology.

It remains to be seen whether this particular instance of sonification will help to consolidate the trustworthiness of science in the public eye, but many sonifications seem well suited to creating a new public engagement with science, beyond a more conventional public understanding of science (Supper 2014: 50). The need for such engagement is particularly acute now that governmental budget cuts increasingly threaten science, the steeply rising expenditure of which is no longer considered self-evident. This situation calls for new and dynamic ways of emphasizing the value of science, and sensory immersion in technologically mediated and artistic inspired sonifications is one such way. It is another, and revealing, ensemble of sonic skills in the sciences. As one would expect, the need for accountability prompted various ways of monitory listening in labs and hospitals - but it also gave a boost to sonifications for wider audiences that tapped into exploratory-synthetic listening through technology-enhanced immersion.

\section{Epilogue}

We experienced an accountability driven and aesthetically informed ensemble of sonic skills ourselves in the aftermath of the Sonic Skills project. When most of the research had been completed, in 2015 we 
organized a Sonic Science Festival with lectures, demonstrations, concerts, a children's workshop, and an exhibition. The festival aimed to present our own research on sound and listening in science, medicine, and engineering, but also to spark the interest of children, young adults, and others in science more generally, through sound and music. For one of the concerts, we collaborated with the Maastricht sound art venue Intro in Situ, and commissioned the young guitar player and composer Aart Strootman to create a piece for his "minimal chamber metal" band Temko. He prepared for his composition by reading the entire The Oxford Handbook of Sound Studies (Pinch and Bijsterveld 2012) and Alexandra Supper's 2014 article on sublime frequencies.

This inspired Strootman to start experimenting with sonification in composition. The first thing he did was to send an email to the US National Aeronautics and Space Administration (NASA). He requested-successfully, to his own surprise-the last set of data that NASA ever received from space probe Pioneer 10 . This spacecraft was launched in 1972 for a mission that would last for over thirty years, until the last moment of contact between NASA and Pioneer 10 on January 22,2003 . At that moment, the distance between Pioneer 10 and the earth was 8 bn kilometers. Strootman's second step was to transform the dataset into the composition Darkness Rises. As well as having the data mold the structure of his musical materials, he expressed time's passing using visually displayed information and radio broadcasts on important events in the political, cultural, scientific, and technological history of humankind. "Sonic Skills paved new paths in my personal composition practice," Strootman concluded. "A scientific approach towards music, in the shape of sonification, has become a ubiquitous component in my writing. 'Darkness Rises' is the first but important step into this territory." 2

The composition immediately attracted the interest of programmers at venues for classical, avantgarde, jazz, and pop music, and was played on eleven occasions. Among them were a concert at the Amsterdam Concertgebouw, a Rotterdam event that showcased Darkness Rises for the Classical: NEXT forum, and a performance in Eindhoven that also featured public intellectual Bas Heijne speaking on the future of science. This last event included the release of Temko's recording of Darkness Rises. But there was more to come: the piece lived on in a version for symphony orchestra. The Philharmonie Zuidnederland programmed Darkness Rises in its Spicy Classics series along with classical music 
concerts "in a modern jacket," a "cross-over" to be enjoyed "with a beer in one's hand," reaching out for new audiences. In its advertising for the concert, the orchestra stressed that the musically rendered dataset "marks the last thing we ... heard from Pioneer $10 \ldots$... It is now travelling through space's gigantic void for eternity." 3

This is a perfect example of rhetorically promising the auditory sublime. What started as our way of bringing science to a wider audience, ended up bringing classical music to new publics with a musical sonification of scientific data. It was the result of a legitimacy exchange par excellence, as music legitimated science's existence while science underscored the vitality of classical music in new formats. The composition was the fruit of an ensemble of sonic skills that we expect to stay around as long as accountability remains important in both the sciences and the arts.

We would have been very unlikely to trace ensembles like this if we had limited our research to historical research alone. Our ethnographies of labs, factories, hospitals, and the sonification community, our reenactments of sound and listening in the sciences, and the long-term relationships we were able to forge with scientists, engineers, doctors, and artists alike were also highly informative. By pairing a sounding history of science with a "sounded anthropology" (Samuels et al. 2010; Bijsterveld 2016), we learned as much about sound in science as about the dynamics between science and technology, science and professions, and science and society. If the history of sound can "disclose previously unknown historical connections," as historian Daniel Morat recapped the ambitions of his peer Mark M. Smith (Morat 2014: 2-3), then our case studies of the sciences through the lens of sound foregrounded the continued significance of trust, and the renewed significance of timing and accountability, in the sciences. They helped us to identify the vitality of the embodied, sound-informed synchronization work required to deal with the tensions between organizational time and expensive instrumental time in today's large-scale laboratories. They enabled us to claim that some sonic skills long ago escaped the trend toward standardization and visualization, or "popped up" again in situations where listening was embedded in legally sanctioned or organizationally invested structures of trust. And they showed us that attempts to invoke the sublime through sonification respond to society's increasing demands for both scientists and artists to account for what they do-by engaging with, rather than conventionally educating, a wide audience. It was a pleasure to listen to them. 


\section{Notes}

1. Personal communication André Kuipers to Karin Bijsterveld, November 29, 2017, courtesy André Kuipers.

2. Personal communication Aart Strootman to Alexandra Supper, January 22, 2017.

3. http://www.philharmoniezuidnederland.nl/concerts/spicy-classics-musicspace/ (last accessed January 31, 2017).

\section{REFERENCES}

Barry, A., Born, G., \& Weszkalnys, G. (2008). Logics of Interdisciplinarity. Economy and Society, 37(1), 20-49.

Bijsterveld, K. (2016). Ethnography and Archival Research in Studying Cultures of Sound. In H. Schulze \& J. Papenburg (Eds.), Sound as Popular Culture: A Research Companion (pp. 99-109). Cambridge: MIT Press.

Born, G., \& Barry, A. (2010). Art-Science: From Public Understanding to Public Experiment. Journal of Cultural Economy, 3(1), 103-119.

Bowker, G. (1993). How to Be Universal: Some Cybernetic Strategies, 19431970. Social Studies of Science, 23(1), 107-127.

Bruyninckx, J. (2017). Synchronicity: Time, Technicians, Instruments, and Invisible Repair. Technology \& Human Values, 42(5), 822-847.

Bruyninckx, J., \& Supper, A. (2016). Sonic Skills in Cultural Contexts: Theories, Practices and Materialities of Listening. Sound Studies: An Interdisciplinary Journal, 2(1), 1-5.

Harris, A. (2016). Listening-Touch, Affect and the Crafting of Medical Bodies Through Percussion. Body \& Society, 22(1), 31-61.

Harris, Y. (2012). Understanding Underwater: The Art and Science of Interpreting Whale Sounds. Interference: A Journal of Audio Culture. Available at http://www.interferencejournal.org/understanding-underwater/. Last accessed August 18, 2017.

Harris, A. (2015). Sounding Disease: Guest Blog at Sociology of Diagnosis Website. Available at https://www.facebook.com/SociologyOfDiagnosis/ posts/799049830181091. Last accessed August 18, 2017.

Harris, A., \& Flynn, E. (2017). Medical Education of Attention: A Qualitative Study of Learning to Listen to Sound. Medical Teacher, 39(1), 79-84.

Hui, A. (2013). The Psychophysical Ear: Musical Experiments, Experimental Sounds, 1840-1910. Cambridge: MIT Press.

Morat, D. (2014). Introduction. In D. Morat (Ed.), Sounds of Modern History: Auditory Cultures in 19th-and 20th-Century Europe (pp. 1-9). New York, NY: Berghahn.

Pellico, L., Duffy, T., Fennie, K., \& Swan, K. (2012). Looking Is Not Seeing and Listening Is Not Hearing: Effect of an Intervention to Enhance Auditory 
Skills of Graduate-Entry Nursing Students. Nursing Education Perspectives, 33(4), 234-239.

Pinch, T., \& Bijsterveld, K. (Eds.). (2012). The Oxford Handbook of Sound Studies. Oxford: Oxford University Press.

Rice, T. (2016). Sounds Inside: Prison, Prisoners and Acoustical Agency. Sound Studies, 2(1), 6-20.

Roosth, S. (2009). Screaming Yeast: Sonocytology, Cytoplasmic Milieus, and Cellular Subjectivities. Critical Inquiry, 35(2), 332-350.

Samuels, D. W., Meintjes, L., Ochoa, A. M., \& Porcello, T. (2010). Soundscapes: Toward a Sounded Anthropology. Annual Review of Anthropology, 39, 329-345.

Supper, A. (2014). Sublime Frequencies: The Construction of Sublime Listening Experiences in the Sonification of Scientific Data. Social Studies of Science, $44(1), 34-58$.

Supper, A. (2015). Data Karaoke: Sensory and Bodily Skills in Conference Presentations. Science as Culture, 24(4), 436-457.

Supper, A. (2016). Lobbying for the Ear, Listening with the Whole Body: The (Anti-)Visual Culture of Sonification. Sound Studies: An Interdisciplinary Journal, 2(1), 69-80.

Open Access This chapter is licensed under the terms of the Creative Commons Attribution-NonCommercial 4.0 International License (http:// creativecommons.org/licenses/by-nc/4.0/), which permits any noncommercial use, sharing, adaptation, distribution and reproduction in any medium or format, as long as you give appropriate credit to the original author(s) and the source, provide a link to the Creative Commons license and indicate if changes were made.

The images or other third party material in this chapter are included in the chapter's Creative Commons license, unless indicated otherwise in a credit line to the material. If material is not included in the chapter's Creative Commons license and your intended use is not permitted by statutory regulation or exceeds the permitted use, you will need to obtain permission directly from the copyright holder.

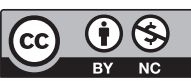

